\title{
Atitudes, Conhecimentos e Perspetivas dos Médicos \\ Portugueses acerca das Terapêuticas Não Convencionais: Um \\ Estudo Transversal
}

\author{
Attitudes, Knowledge and Views of Portuguese Physicians \\ regarding Complementary and Alternative Medicine: A Cross- \\ -Sectional Study
}

Carlos NOGUEIRA $\triangle^{1}$, Armando BRITO DE SÁ2, Ana Paula MARTINS ${ }^{3}$
Acta Med Port 2022 May;35(5):320-327 $\cdot$ https://doi.org/10.20344/amp.16073

\begin{abstract}
RESUMO
Introdução: A utilização significativa das terapêuticas não convencionais, e o facto de estas não serem inócuas, torna imperativo que os médicos as conheçam, de forma a melhor aconselharem os seus doentes. Este estudo visou identificar as atitudes, conhecimentos e perspetivas dos médicos portugueses relativamente às terapêuticas não convencionais.

Material e Métodos: Realizou-se um estudo observacional, analítico e transversal. Os médicos inscritos na Ordem dos Médicos portuguesa responderam a um questionário com respostas em escala de Likert de cinco pontos, dicotómicas e de escolha múltipla. As respostas às questões sobre crenças/convicções a respeito das terapêuticas não convencionais; aspetos relacionados com as terapêuticas não convencionais aprovadas pela Lei portuguesa; fontes usadas para obtenção de informação relativamente às terapêuticas não convencionais; e perguntas sobre as terapêuticas não convencionais no contexto nacional, foram analisadas com recurso ao SPSS v.27.0. Todas as conclusões foram tomadas ao nível de significância de $5 \%$.

Resultados: Dos 4334 médicos que responderam na íntegra ao questionário, 45,5\% não se sentem confortáveis para conversar, argumentar e esclarecer os doentes a respeito das terapêuticas não convencionais. A maioria, $68,6 \%$, considera que a competência em acupuntura da Ordem dos Médicos deveria permanecer disponível. No entanto, $72,4 \%$ dos médicos consideram que as terapêuticas não convencionais não deveriam ser incluídas no Serviço Nacional de Saúde.
\end{abstract}

Conclusão: Os conhecimentos dos médicos portugueses relativamente às terapêuticas não convencionais parecem ser reduzidos, sendo que parte relevante dos inquiridos não se considera preparada para aconselhar os seus doentes.

Palavras-chave: Educação Médica; Portugal; Terapias Complementares

\section{ABSTRACT}

Introduction: The widespread use of complementary and alternative medicine and the fact that these are not innocuous makes it important for physicians to be familiar with it in order to properly advise their patients. The aim of this study was to identify attitudes, knowledge and views of Portuguese physicians about complementary and alternative medicine.

Material and Methods: An observational, analytical, cross-sectional study was performed. A questionnaire with dichotomous and multiple-choice answers on a 5-point Likert scale, was sent to all doctors registered in the Portuguese Medical Association. It included beliefs and convictions regarding complementary and alternative medicine; aspects related to the types of complementary and alternative medicine legally approved in Portugal; sources used to obtain information about complementary and alternative medicine; and questions about complementary and alternative medicine in the Portuguese context. The statistical analysis of the data was made using SPSS v. 27.0 and all conclusions were taken at a significance level of $5 \%$.

Results: From a total sample of 4334 doctors that fully completed the questionnaire, $45.5 \%$ reported not feeling comfortable talking, debating, and elucidating their patients about complementary and alternative medicine. The majority, $68.6 \%$, considered that the competence in medical acupuncture of the Portuguese Medical Association should remain available. However, $72.4 \%$ believe that complementary and alternative medicine should not be included in the National Health Service.

Conclusion: Portuguese doctors seem to have a low level of knowledge about complementary and alternative medicine, and a relevant part of them does not feel prepared to advise their patients about it.

Keywords: Complementary Therapies; Education, Medical; Portugal

\section{INTRODUÇÃO}

As expressões 'medicina tradicional', 'medicina não convencional' ou 'medicina complementar e alternativa' são usadas quase indistintamente para se referir ao conjunto de diversos elementos, práticas ou produtos relacionados com cuidados de saúde que geralmente não fazem parte da medicina dita convencional. ${ }^{1}$ Em Portugal, "terapêuticas não convencionais" (TNC) é a terminologia oficialmente reconhecida pelo estado. De acordo com a Lei n45/2003, que iniciou a legislação e o enquadramento destas práticas, "consideram-se terapêuticas não convencionais aquelas que partem de uma base filosófica diferente da medicina convencional e aplicam processos específicos de diagnóstico e terapêuticas próprias". ${ }^{2}$

Existe uma panóplia enorme de terapêuticas não

1. Faculdade de Ciências da Saúde. Universidade da Beira Interior. Covilhã. Portugal

2. Unidade de Saúde Familiar Conde Saúde. Agrupamento de Centros de Saúde da Arrábida. Administração Regional de Saúde de Lisboa e Vale do Tejo. Sesimbra. Portugal.

3. Departamento de Matemática. Centro de Matemática e Aplicações. Universidade da Beira Interior. Covilhã. Portugal.

$\triangle$ Autor correspondente: Carlos Nogueira. a35383@fcsaude.ubi.pt

Recebido/Received: 02/03/2021 - Aceite/Accepted: 06/08/2021 - Publicado Online/Published Online: 02/02/2022 - Publicado/Published: 02/05/2022

Copyright $\odot$ Ordem dos Médicos 2022 
convencionais, tais como reflexologia, reiki, medicina ayurveda, aromaterapia, cristaloterapia, urinoterapia, ozonoterapia, entre outras. No entanto, a medicina tradicional chinesa, a fitoterapia, a homeopatia, a acupuntura, a naturopatia, a osteopatia e a quiropraxia representam as terapêuticas aprovadas pela Lei portuguesa. ${ }^{3,4}$

As TNC detêm uma imensa popularidade a nível mundial. Um relatório do National Center for Health Statistics (NCHS) estimou que, em 2012, 34\% dos americanos adultos e $12 \%$ das crianças americanas recorreram a TNC.5,6 Em 2007, 38,1 milhões de americanos gastaram aproximadamente 33,9 mil milhões de dólares em consultas e produtos relacionados com as terapêuticas não convencionais. ${ }^{7}$ Na Europa, cerca de $70 \%$ dos cidadãos utilizam-nas ou já as utilizaram e de acordo com o relatório do Observatório Europeu de Sistemas e Políticas de Saúde sobre Portugal, estima-se que mais de dois milhões de cidadãos procuram regularmente as TNC. 8,9

Apesar da sua popularidade, as TNC não são consensuais entre a comunidade médica e científica. $O$ fator que mais contribui para a controvérsia é a carência de evidência científica suficientemente válida e robusta para comprovar a eficácia das terapêuticas não convencionais. ${ }^{10-14}$ Para além disso, a falta de regulação legal destas práticas abre espaço para o exercício fraudulento, erros e atrasos de diagnósticos, informações erradas e tratamentos nocivos para os doentes. ${ }^{4} \mathrm{O}$ atraso ou o abandono dos tratamentos convencionais, os efeitos adversos diretos de algumas práticas e a interação com tratamentos farmacológicos convencionais estão entre as principais causas dos casos danosos descritos na literatura. ${ }^{15,16}$

Devido à utilização significativa das TNC em diversos países e ao facto de não serem inócuas, é imperativo que os profissionais de saúde em geral e os médicos em particular, como agentes primordiais na comunicação com o doente, tenham conhecimento básico das TNC de forma a aconselharem e orientarem apropriadamente os seus doentes. ${ }^{17}$

Apesar de conteúdos básicos relativos às TNC serem atualmente abordados em algumas faculdades de medicina, esta temática não se vê representada de forma relevante nos currículos de formação médica. Prova disso, são os diversos estudos realizados que reportam que a maioria dos médicos sente que o seu conhecimento relativamente à segurança e eficácia das TNC é inadequado e que mais treino e educação são necessários. ${ }^{18-21}$

Os Estados Unidos, México, Reino Unido, Alemanha, Polónia e Suécia, estão entre as muitas nações em que já foram realizados estudos com o objetivo de entender qual a opinião e o nível de conhecimento médico relativamente às terapêuticas não convencionais. ${ }^{17-24} \mathrm{Em}$ Portugal, apenas temos conhecimento de um estudo sobre a temática em que foram avaliadas as perspetivas dos médicos portugueses de medicina geral e familiar. ${ }^{25}$ Nesse sentido, o presente trabalho teve como objetivo identificar as atitudes, conhecimentos e perspetivas dos médicos portugueses relativamente às TNC legalmente reguladas em Portugal.
Para além disso, nos estudos realizados internacionalmente, o sexo e a idade são destacados como fatores que influenciam a opinião dos médicos relativamente às TNC, pelo que a influência dessas variáveis também configurou um objetivo desta investigação.

\section{MATERIAL E MÉTODOS}

Realizou-se um estudo primário, observacional, analítico e transversal, com aplicação de um questionário construído especificamente para medir as atitudes, conhecimentos e perspetivas dos médicos portugueses relativamente às TNC.

A população-alvo do estudo foi constituída pelos médicos inscritos na Ordem dos Médicos portuguesa, que colaborou no envio dos questionários aos seus inscritos. No final do ano de 2019, estavam inscritos 56200 médicos na Ordem dos Médicos, não nos sendo possível saber quantos destes estavam incluídos na lista de endereços eletrónicos da Ordem dos Médicos.

O estudo tomou a forma de um questionário auto-preenchido. A sua elaboração teve duas fases. Numa fase inicial, procedeu-se a uma revisão da literatura científica relativamente às diversas terapêuticas não convencionais. Para além disso, efetuou-se uma pesquisa em bases de dados médicas, nacionais e internacionais, de trabalhos que envolvessem as perspetivas da comunidade médica relativamente às $\mathrm{TNC}$.

$\mathrm{Na}$ fase seguinte, analisaram-se os questionários usados em outros trabalhos. Não tendo sido encontrados questionários padronizados e validados que pudessem ser usados de forma integral, atendendo aos objetivos deste estudo, procedeu-se à construção de um questionário (Apêndice 1: https://www.actamedicaportuguesa.com/revista/index.php/amp/article/view/16063/Apendice_01.pdf), seguindo as linhas orientadoras das investigações internacionais realizadas no mesmo âmbito. Deu-se ênfase à criação de perguntas originais que se julgam adaptadas à realidade nacional. ${ }^{18,23,25,26}$

O questionário é composto por questões com respostas em escala de Likert de cinco pontos, dicotómicas e de escolha múltipla, estando dividido em seis partes: uma apresentação breve da investigação, o consentimento informado e quatro secções (A, B, C e D). As questões sociodemográficas integram a secção $A$. A secção $B$, contempla perguntas relativas às crenças/convicções dos médicos portugueses a respeito das TNC. As questões relacionadas com as TNC aprovadas pela lei portuguesa, bem como perguntas relativas às fontes usadas pelos inquiridos para obtenção de informação relativamente às TNC, formam a secção $C$. Finalmente, a secção $D$ abrange perguntas mais específicas sobre as TNC no contexto nacional.

O questionário foi informatizado com a tecnologia LimeSurvey ${ }^{\circledR}$ e disponibilizado por e-mail para a mailing list da Ordem dos Médicos pelos seus serviços, sem interferência dos investigadores neste processo. O questionário esteve disponível online entre os dias 12 de agosto de 2020 e 4 de setembro de 2020. 
Para análise estatística dos dados recolhidos foi usado o software SPSS v. 27.

$\mathrm{Na}$ análise descritiva da amostra apresentam-se tabelas de frequências e gráficos ilustrativos das distribuições das diversas variáveis estatísticas. Para as variáveis estatísticas quantitativas ou medidas em escala de Likert, apresenta-se a média (e.g. para as questões numa escala de 1 a 5 , um valor superior a 3 é superior ao ponto intermédio da escala), o desvio padrão, o mínimo e o máximo.

$\mathrm{Na}$ análise inferencial, embora estivéssemos a lidar com escalas de Likert, ou seja, com dados em escala ordinal, seguimos a abordagem de Sullivan e Artino ${ }^{27}$ que justificam que os testes paramétricos não só podem ser usados com dados ordinais, como são neste caso mais robustos que os não paramétricos, mesmo quando a hipótese de normalidade é violada. Na comparação de duas ou de mais médias populacionais aplicou-se, respetivamente, o teste paramétrico $t$ Student e a análise de variância (ANOVA).

Com base nas tabelas de contingência, construiu-se o teste de homogeneidade do qui-quadrado para verificar se duas ou mais populações são homogéneas entre si, ou seja, para testar se as proporções de elementos com determinadas características são iguais para todas as populações. ${ }^{28}$

Para além das estimativas pontuais apresentam-se os intervalos de confiança a $95 \%$ e considera-se uma probabilidade de erro de primeira espécie de $5 \%$ nas restantes inferências para a população de médicos portugueses.

O presente estudo obteve o parecer favorável da Comissão de Ética da Universidade da Beira Interior. Assegurou-se o anonimato dos médicos inquiridos, bem como a confidencialidade dos dados recolhidos.

\section{RESULTADOS}

Na amostra de médicos considerada ( $n=4334), 52,8 \%$ (2287) são do sexo feminino e os restantes 47,2\% (2047) são do sexo masculino. Apresentam uma idade média de 46,8 anos, com um desvio padrão de 15,7 anos, tendo o médico mais novo 23 anos e o mais velho 98 anos. Duas idades foram mal reportadas e por isso não foram consideradas. A idade mediana foi de 43 anos, apresentando a distribuição de frequências da idade uma assimetria positiva (coeficiente de assimetria igual a 0,332, com um erro padrão de 0,037 ). As especialidades médicas mais representadas são Medicina Geral e Familiar (22,4\%), Medicina Interna (6,9\%), Pediatria (4,6\%) e Anestesiologia (4,6\%), destacando-se $17,7 \%$ sem especialidade.

Nas questões sobre as convicções relativas às TNC os itens com uma concordância média (M) superior ao ponto intermédio da escala de medida são o "3 - as crenças, valores e expectativas dos doentes deverão ser integrados na prestação de cuidados médicos" ( $M=4,12)$ e "6 - os tratamentos não testados cientificamente de forma rigorosa deverão ser desencorajados" ( $M=4,00)$, seguidas de "5 - os efeitos das terapêuticas não convencionais são essencialmente resultado do efeito placebo" $(M=3,43)$ e depois de "7 - as terapêuticas não convencionais incluem ideias e métodos que poderiam ser utilizados de forma benéfica pela medicina convencional" ( $M=3,26)$ e "4 - as terapêuticas não convencionais são uma ameaça à saúde pública" $(M=3,18)$. Finalmente, os itens "1 - a saúde física e mental é mantida por uma energia ou força vital" $(M=2,76)$ e "2 - o corpo do ser humano é essencialmente auto-curativo sendo a tarefa do médico providenciar a harmonia necessária para esse processo" ( $\mathrm{M}=2,71$ ) apresentam uma concordância média inferior ao ponto intermédio da escala de medida (Tabela 1).

A concordância com a afirmação «A saúde física e mental é mantida por uma energia ou força vital» é superior para o sexo feminino $(M=3,00)$ e inferior para o sexo masculino $(M=2,48)$, enquanto a concordância com a afirmação "Os efeitos das terapias não convencionais são essencialmente resultado do efeito placebo» é superior para o sexo masculino $(M=3,63)$ e inferior para o sexo feminino $(M=3,24)$. Em ambos os casos as diferenças são estatisticamente significativas $[t(4149,742)=14,218, p<0,001$, $I_{95 \%}=(0,446 ; 0,589)$ e $t(4332)=-11,787, p<0,001, I C_{95 \%}$ $=(-0,456 ;-0,326)]$ apesar da diferença entre as médias ser estimada em menos de meio ponto na escala de 1 a 5 (Tabela 1).

Tendo em conta três classes etárias para a idade dos médicos: até 35 anos ( $\mathrm{n}=1469,33,9 \%), 36-55$ anos $(\mathrm{n}=$ $1316,30,4 \%$ ) e mais de 55 anos ( $n=1547,35,7 \%$ ), a concordância média com a afirmação "a saúde física e mental é mantida por uma energia ou força vital» é inferior para idades até 35 anos $(M=2,47)$, seguidos de $36-55$ anos ( $M$ $=2,74)$ e superior para mais de 55 anos $(M=3,04)$, sendo estas diferenças estatisticamente significativas [F (2;4329) $=86,682, p<0,001]$. Contrariamente, na afirmação «os efeitos das terapias não convencionais são essencialmente resultado do efeito placebo» a concordância média é superior para idades até 35 anos $(M=3,71)$, seguidos de $36-55$ anos $(M=3,40)$ e inferior para mais de 55 anos $(M=3,18)$, sendo também as diferenças estatisticamente significativas $[F(2 ; 4329)=88,208, p<0,001]$ (Tabela 1).

O grau de conhecimento das TNC dos médicos portugueses é superior para a acupuntura $\left[\mathrm{M}=3,29\right.$; $\mathrm{IC}_{95 \%}$ $=(3,26 ; 3,32)]$, única terapêutica com um conhecimento médio superior ao ponto intermédio da escala de medida. Segue-se a osteopatia $\left[\mathrm{M}=2,76 ; \mathrm{IC}_{95 \%}=(2,72 ; 2,79)\right]$ e homeopatia $\left[\mathrm{M}=2,72 ; \mathrm{IC}_{95 \%}=(2,68 ; 2,75)\right]$. O menor conhecimento reporta-se à quiropraxia $\left[\mathrm{M}=1,97 ; \mathrm{IC}_{95 \%}=(1,94\right.$; 2,01)] (Tabela 2).

A perceção de eficácia das TNC dos médicos portugueses é superior para acupuntura $\left[\mathrm{M}=3,24 ; \mathrm{IC}_{95 \%}=(3,20\right.$; $3,27)$ ], única terapêutica com uma perceção de eficácia média superior ao ponto intermédio da escala de medida, sendo inferior para naturopatia $\left[\mathrm{M}=1,84 ; \mathrm{IC}_{95 \%}=(1,80 ; 1,88)\right] \mathrm{e}$ homeopatia $\left[\mathrm{M}=1,76 ; \mathrm{IC}_{95 \%}=(1,73 ; 1,80)\right]$ (Tabela 3).

Os três fatores que mais influenciam as convicções dos médicos relativamente às terapêuticas não convencionais são os "testemunhos de doentes" $\left[46,3 \%, \mathrm{IC}_{95 \%}=(44,8 \%\right.$; $47,8 \%)$ ], seguido de "opiniões de colegas profissionais de saúde" $\left[43,2 \%, I C_{95 \%}=(41,7 \% ; 44,7 \%)\right]$ e "revisões 
Tabela 1 - Secção B: Relativamente às seguintes afirmações, qual o seu grau de concordância de acordo com as suas convicções?

\begin{tabular}{|c|c|c|c|c|c|c|c|}
\hline & 1 & 2 & 3 & 4 & 5 & & \\
\hline & n (\%) & n (\%) & n (\%) & n (\%) & n (\%) & $M(D P)$ & $p$ \\
\hline $\begin{array}{l}1 \text { - A saúde física e mental é mantida por } \\
\text { uma energia ou força vital. }\end{array}$ & $899(20,7)$ & $901(20,8)$ & $1171(27,0)$ & $1087(25,1)$ & $276(6,4)$ & $2,76(1,22)$ & \\
\hline Feminino & $280(12,2)$ & $461(20,2)$ & $691(30,2)$ & $689(30,1)$ & $166(7,3)$ & $3,0(1,13)$ & $<0,001$ \\
\hline Masculino & $619(30,2)$ & $440(21,5)$ & $480(23,4)$ & $398(19,4)$ & $110(5,4)$ & $2,48(1,25)$ & \\
\hline Até 35 anos & $420(28,6)$ & $343(23,3)$ & $366(24,9)$ & $278(18,9)$ & $62(4,2)$ & $2,47(1,21)$ & $<0,001$ \\
\hline $36-55$ anos & $274(20,8)$ & $268(20,4)$ & $387(29,4)$ & $301(22,9)$ & $86(6,5)$ & $2.74(1,21)$ & \\
\hline Mais de 55 anos & $205(13,3)$ & $289(18,7)$ & $418(27,0)$ & $508(32,8)$ & $127(8,2)$ & $3,04(1,17)$ & \\
\hline $\begin{array}{l}2 \text { - O corpo do ser humano é } \\
\text { essencialmente autocurativo sendo a } \\
\text { tarefa do médico providenciar a harmonia } \\
\text { necessária para esse processo. }\end{array}$ & $646(14,9)$ & $1485(34,3)$ & $866(20,0)$ & $1164(26,9)$ & $173(4,0)$ & $2,71(1,13)$ & \\
\hline $\begin{array}{l}3 \text { - As crenças, valores e expectativas } \\
\text { dos doentes deverão ser integrados na } \\
\text { prestação de cuidados médicos. }\end{array}$ & $61(1,4)$ & $175(4,0)$ & $358(8,3)$ & $2324(53,6)$ & $1416(32,7)$ & $4,12(0,83)$ & \\
\hline $\begin{array}{l}4 \text { - As terapêuticas não convencionais são } \\
\text { uma ameaça à saúde pública. }\end{array}$ & $290(6,7)$ & $1113(25,7)$ & $1098(25,3)$ & $1194(27,5)$ & $639(14,7)$ & $3,18(1,17)$ & \\
\hline $\begin{array}{l}5 \text { - Os efeitos das terapêuticas não } \\
\text { convencionais são essencialmente } \\
\text { resultado do efeito placebo. }\end{array}$ & $160(3,7)$ & $907(20,9)$ & $924(21,3)$ & $1603(37,0)$ & $740(17,1)$ & $3,43(1,11)$ & \\
\hline Feminino & $98(4,3)$ & $574(25,1)$ & $552(24,1)$ & $799(34,9)$ & $264(11,5)$ & $3,24(1,08)$ & $<0,001$ \\
\hline Masculino & $62(3,0)$ & $333(16,3)$ & $372(18,2)$ & $804(39,3)$ & $476(23,3)$ & $3,63(1,10)$ & \\
\hline Até 35 anos & $28(1,9)$ & $195(13,3)$ & $301(20,5)$ & $599(40,8)$ & $346(23,6)$ & $3,71(1,03)$ & $<0,001$ \\
\hline $36-55$ anos & $50(3,8)$ & $285(21,7)$ & $294(22,3)$ & $461(35,0)$ & $226(17,2)$ & $3,40(1,12)$ & \\
\hline Mais de 55 anos & $82(5,3)$ & $427(27,6)$ & $329(21,3)$ & $542(65,0)$ & $167(10,8)$ & $3,18(1,11)$ & \\
\hline $\begin{array}{l}6 \text { - Os tratamentos não testados } \\
\text { cientificamente de forma rigorosa deverão } \\
\text { ser desencorajados. }\end{array}$ & $68(1,6)$ & $405(9,3)$ & $587(13,5)$ & $1688(38,9)$ & $1586(36,6)$ & $4,00(1,01)$ & \\
\hline $\begin{array}{l}7 \text { - As terapêuticas não convencionais } \\
\text { incluem ideias e métodos que poderiam ser } \\
\text { utilizados de forma benéfica pela medicina } \\
\text { convencional. }\end{array}$ & $323(7,5)$ & $766(17,7)$ & $1071(24,7)$ & $1820(42,0)$ & $354(8,2)$ & $3,26(1,07)$ & \\
\hline $\begin{array}{l}8 \text { - As terapêuticas não convencionais são } \\
\text { apenas um embuste com fins financeiros. }\end{array}$ & $283(6,5)$ & $1252(28,9)$ & $1263(29,1)$ & $1039(24,0)$ & $497(11,5)$ & $3,05(1,12)$ & \\
\hline
\end{tabular}

Os valores indicados reportam-se à escala de medida: 1- Discordo totalmente; 2- Discordo; 3- Não concordo nem discordo; 4- Concordo; 5- Concordo totalmente.

M: média; DP: desvio padrão

sistemáticas/metanálises" $\left[42,1 \%, \mathrm{IC}_{95 \%}=(40,6 \% ; 43,6 \%)\right]$.

Estimou-se em $68,6 \%\left[\mathrm{IC}_{95 \%}=(67,2 ; 70,0 \%)\right]$ a percentagem de médicos que considera que a competência em acupuntura médica da Ordem dos Médicos deveria permanecer disponível. Esta percentagem é superior para o sexo feminino $(74,3 \%)$ e inferior para o sexo masculino $(62,2 \%)$, acontecendo o inverso com a opinião que deveria ser extinta, sendo as diferenças observadas estatisticamente significativas [teste do qui-quadrado, $\chi(1)=72,835 ; p<0,001$ ]. A percentagem que considera que a competência em acupuntura médica da Ordem dos Médicos deveria ser extinta é superior para idades até 35 anos (44,6\%), seguidas de 36 - 55 anos (34,3\%) e inferior para mais de 55 anos (16,4\%), acontecendo o inverso com a opinião sobre se deveria permanecer disponível, sendo as diferenças observadas estatisticamente significativas [teste do qui-quadrado, $\chi(2)=$ 286,368; $p<0,001]$.

Verificou-se que $45,5 \%$ [IC $\left.{ }_{95 \%}=(44,0 \% ; 47,0 \%)\right]$ dos mé- dicos não se sente confortável para conversar, argumentar e esclarecer os seus doentes a respeito dais,s terapêuticas não convencionais, $57,0 \%$ [ $\mathrm{IC}_{95 \%}=(55,5 \%$; 58,5\%) $]$ considera que os currículos de formação médica deveriam incluir mais conteúdos sobre terapêuticas não convencionais, $29,3 \%\left[\mathrm{IC}_{95 \%}=(27,9 \% ; 30,6 \%)\right]$ já referenciou pelo menos uma vez um doente para um profissional de terapêuticas não convencionais e $72,4 \%$ [ $\left.\mathrm{IC}_{95 \%}=(71,1 \% ; 73,7 \%)\right]$ considera que as terapêuticas não convencionais não deveriam ser incluídas no Serviço Nacional de Saúde (Tabela 4).

A percentagem que considera que se sente confortável para conversar, argumentar e esclarecer os seus doentes a respeito das terapêuticas não convencionais é superior para o sexo masculino $(69,4 \%)$ e inferior para o sexo feminino $(41,2 \%)$, sendo as diferenças observadas estatisticamente significativas [teste do qui-quadrado, $\chi(1)=345,926$; $p<0,001]$. As diferenças observadas em relação aos grupos etários também são estatisticamente significativas 
Tabela 3 - Questão: “10 - Relativamente à sua perceção de eficácia das seguintes terapêuticas não convencionais, assinale:”

\begin{tabular}{lcccccccc}
\hline & Não sei & $\mathbf{1}$ & $\mathbf{2}$ & $\mathbf{3}$ & $\mathbf{4}$ & $\mathbf{5}$ & \\
\cline { 2 - 7 } & $\mathrm{n}(\%)$ & $\mathrm{n}(\%)$ & $\mathrm{n}(\%)$ & $\mathrm{n}(\%)$ & $\mathrm{n}(\%)$ & $\mathrm{n}(\%)$ & M (DP) & \multicolumn{1}{c}{ IC $\mathbf{9 5}_{\mathbf{9 5}}$} \\
\hline Acupuntura & $283(6,5)$ & $316(7,3)$ & $534(12,3)$ & $1222(28,2)$ & $1840(42,5)$ & $139(3,2)$ & $3,24(0,99)$ & $(3,20 ; 3,27)$ \\
Homeopatia & $929(21,4)$ & $2067(47,7)$ & $470(10,8)$ & $508(11,7)$ & $326(7,5)$ & $34(0,8)$ & $1,76(1,08)$ & $(1,73 ; 1,80)$ \\
Naturopatia & $1827(42,2)$ & $1339(30,9)$ & $505(11,7)$ & $404(9,3)$ & $240(5,5)$ & $19(0,4)$ & $1,84(1,06)$ & $(1,80 ; 1,88)$ \\
Osteopatia & $997(23,0)$ & $588(13,6)$ & $565(13,0)$ & $940(21,7)$ & $1119(25,8)$ & $125(2,9)$ & $2,89(1,16)$ & $(2,85 ; 2,93)$ \\
Medicina tradicional chinesan & $1632(37,7)$ & $840(19,4)$ & $528(12,2)$ & $651(15,0)$ & $626(14,4)$ & $57(1,3)$ & $2,46(1,21)$ & $(2,41 ; 2,50)$ \\
Fitoterapia & $1973(45,5)$ & $985(22,7)$ & $460(10,6)$ & $452(10,4)$ & $402(9,3)$ & $62(1,4)$ & $2,19(1,22)$ & $(2,14 ; 2,24)$ \\
Quiropraxia & $2341(54,0)$ & $860(19,8)$ & $369(8,5)$ & $405(9,3)$ & $331(7,6)$ & $28(0,6)$ & $2,15(1,19)$ & $(2,09 ; 2,20)$ \\
\hline
\end{tabular}

Os valores indicados reportam-se à escala de medida: 1- Nada eficaz; 2- Muito pouco eficaz; 3- Pouco eficaz; 4- Eficaz; 5- Extremamente eficaz.

M: média; DP: desvio padrão; $\mathrm{IC}_{95 \%}$ : intervalo de confiança $95 \%$ para a média.

Tabela 2 - Questão: "9 - Relativamente ao seu grau de conhecimento das seguintes terapêuticas não convencionais, assinale:"

\begin{tabular}{|c|c|c|c|c|c|c|c|}
\hline & 1 & 2 & 3 & 4 & 5 & & \\
\hline & $\mathrm{n}(\%)$ & $\mathrm{n}(\%)$ & $\mathrm{n}(\%)$ & $\mathrm{n}(\%)$ & $\mathrm{n}(\%)$ & M (DP) & IC $_{95 \%}$ \\
\hline Acupuntura & $269(6,2)$ & $827(19,1)$ & $967(22,3)$ & $1923(44,4)$ & $348(8,0)$ & $3,29(1,06)$ & $(3,26 ; 3,32)$ \\
\hline Homeopatia & $911(21,0)$ & $1074(24,8)$ & $928(21,4)$ & $1169(27,0)$ & $252(5,8)$ & $2,72(1,23)$ & $(2,68 ; 2,75)$ \\
\hline Naturopatia & $1636(37,7)$ & $1116(25,7)$ & $831(19,2)$ & $648(15,0)$ & $103(2,4)$ & $2,18(1,16)$ & $(2,15 ; 2,22)$ \\
\hline Osteopatia & $849(19,6)$ & $1063(24,5)$ & $921(21,3)$ & $1294(29,9)$ & $207(4,8)$ & $2,76(1,21)$ & $(2,72 ; 2,79)$ \\
\hline Medicina tradicional chinesa & $1223(28,2)$ & $1184(27,3)$ & $918(21,2)$ & $904(20,9)$ & $105(2,4)$ & $2,42(1,17)$ & $(2,38 ; 2,45)$ \\
\hline Fitoterapia & $1610(37,1)$ & $1088(25,1)$ & $783(18,1)$ & $702(16,2)$ & $151(3,5)$ & $2,24(1,21)$ & $(2,20 ; 2,27)$ \\
\hline Quiropraxia & $2066(47,7)$ & $999(23,1)$ & $671(15,5)$ & $520(12,0)$ & $78(1,8)$ & $1,97(1,13)$ & $(1,94 ; 2,01)$ \\
\hline
\end{tabular}

Os valores indicados reportam-se à escala de medida: 1- Nenhum; 2- Muito pouco; 3- Pouco; 4- Algum; 5- Muito.

M: média; DP: desvio padrão; IC $_{95 \%}$ : intervalo de confiança $95 \%$ para a média.

[teste do qui-quadrado, $\chi(2)=9,292 ; p=0,010$ ] (Tabela 4).

No que concerne à referenciação de um doente para um profissional de terapêuticas não convencionais, $31,4 \%$ dos médicos do sexo feminino e $27,1 \%$ do sexo masculino, já referenciaram, sendo estas diferenças estatisticamente significativas [teste do qui-quadrado, $\chi(1)=9,579 ; p=$ 0,002 ] (Tabela 4). A referenciação foi inferior para idades até 35 anos $(12,7 \%)$, seguidos de $36-55$ anos $(30,9 \%)$ e superior para mais de 55 anos (43,9\%), sendo as diferenças observadas estatisticamente significativas [teste do qui-quadrado, $\chi(2)=356,557 ; p<0,001$ ] (Tabela 4).

Apenas $34,3 \%$ dos médicos do sexo feminino e $20,1 \%$ do sexo masculino considera que as terapêuticas não convencionais deveriam ser incluídas no Serviço Nacional de Saúde. As diferenças observadas entre os sexos [teste do qui-quadrado, $\chi(1)=109,010 ; p<0,001]$ e entre os grupos etários são estatisticamente significativas [teste do qui-quadrado, $\chi^{2}(2)=103,633 ; p<0,001$ ] (Tabela 4).

A concordância média com a afirmação "Os efeitos das terapias não convencionais são essencialmente resultado do efeito placebo» é inferior para quem considera que terapêuticas não convencionais deveriam ser incluídas no Serviço Nacional de Saúde $(M=2,43)$ e superior para quem não considera $(M=3,81)$, sendo as diferenças estatisticamente significativas $\left[t(4332)=-43,939, p<0,001, I C_{95 \%}=\right.$ $(-1,437 ;-1,314)]$. A concordância média com a afirmação «os tratamentos não testados cientificamente de forma rigorosa, deverão ser desencorajados» é inferior para quem considera que terapêuticas não convencionais deveriam ser incluídas no Serviço Nacional de Saúde $(M=3,28)$ e superior para quem não considera ( $M=4,27)$, sendo as diferenças estatisticamente significativas $[t(1771,775)=$ $\left.-28,995, p<0,001, I C_{95 \%}=(-1,064 ;-0,929)\right]$. A concordância média com a afirmação «as terapêuticas não convencionais incluem ideias e métodos que poderiam ser utilizados de forma benéfica pela medicina convencional» é superior para quem considera que terapêuticas não convencionais deveriam ser incluídas no Serviço Nacional de Saúde (SNS) ( $M=4,07)$ e inferior para quem não considera ( $M$ $=2,95)$, sendo as diferenças estatisticamente significativas $\left[t(43345,228)=42,160, p<0,001, I_{95 \%}=(1,074 ; 1,178)\right]$ (Tabela 5).

A concordância média com a afirmação «a saúde física e mental é mantida por uma energia ou força vital» é superior para quem considera que a competência em acupuntura médica da Ordem dos Médicos deveria permanecer disponível $(M=3,10)$ e inferior para quem considera que deveria ser extinta ( $M=2,00)$, sendo as diferenças estatisticamente significativas $\left[t(4332)=30,467, p<0,001, I_{95 \%}\right.$ $=(1,031 ; 1,173)]$. Em relação à afirmação «os tratamentos não testados cientificamente de forma rigorosa deverão ser desencorajados» a concordância média é superior para quem considera que a competência em acupuntura médica da Ordem dos Médicos deveria ser extinta $(M=4,54)$ e inferior para quem considera que deveria permanecer disponível $(M=3,75)$, sendo as diferenças estatisticamente significativas $\left[t(3773,122)=-29,596, p<0,001,1 C_{95 \%}=\right.$ $(-0,840 ;-0,736)]$ (Tabela 6). 
Tabela 4 - Estatísticas e teste do qui-quadrado, relação entre o género, idade e as questões 13,14, 15 e 16

\begin{tabular}{|c|c|c|c|}
\hline & Não & Sim & \\
\hline & $\mathrm{n}(\%)$ & $\mathrm{n}(\%)$ & $p$ \\
\hline $\begin{array}{l}13 \text { - Sente-se confortável para conversar, argumentar e esclarecer os seus doentes a } \\
\text { respeito das terapêuticas não convencionais? }\end{array}$ & $1972(45,5)$ & $2362(54,5)$ & $<0,001$ \\
\hline Feminino & $1345(58,8)$ & $942(41,2)$ & $<0,001$ \\
\hline Masculino & $627(30,6)$ & $1420(69,4)$ & \\
\hline Até 35 anos & $698(47,5)$ & $771(52,5)$ & 0,010 \\
\hline $36-55$ anos & $616(46,8)$ & $700(53,2)$ & \\
\hline Mais de 55 anos & $656(42,4)$ & $891(57,6)$ & \\
\hline $\begin{array}{l}14 \text { - Considera que os currículos de formação médica deveriam incluir mais conteúdos } \\
\text { sobre terapêuticas não convencionais? }\end{array}$ & $1863(43,0)$ & $2471(57,0)$ & $<0,001$ \\
\hline Feminino & $809(35,4)$ & $1478(64,6)$ & $<0,001$ \\
\hline Masculino & $1054(51,5)$ & $993(48,5)$ & \\
\hline Até 35 anos & $767(52,2)$ & $702(47,8)$ & $<0,001$ \\
\hline $36-55$ anos & $600(45,6)$ & $716(54,4)$ & \\
\hline Mais de 55 anos & $495(32,0)$ & $1052(68,0)$ & \\
\hline $\begin{array}{l}15 \text { - Alguma vez referenciou um doente para um profissional de terapêuticas não } \\
\text { convencionais? }\end{array}$ & $3063(70,7)$ & $1271(29,3)$ & $<0,001$ \\
\hline Feminino & $1570(68,6)$ & $717(31,4)$ & 0,002 \\
\hline Masculino & $1493(72,9)$ & $554(27,1)$ & \\
\hline Até 35 anos & $1283(87,3)$ & $186(12,7)$ & $<0,001$ \\
\hline $36-55$ anos & $910(69,1)$ & $406(30,9)$ & \\
\hline Mais de 55 anos & $868(56,1)$ & $679(43,9)$ & \\
\hline $\begin{array}{l}16 \text { - As terapêuticas não convencionais deveriam ser incluídas no Serviço Nacional de } \\
\text { Saúde? }\end{array}$ & $3138(72,4)$ & $1196(27,6)$ & $<0,001$ \\
\hline Feminino & $1502(65,7)$ & $785(34,3)$ & $<0,001$ \\
\hline Masculino & $1636(79,9)$ & $411(20,1)$ & \\
\hline Até 35 anos & $1194(81,3)$ & $275(18,7)$ & $<0,001$ \\
\hline $36-55$ anos & $940(71,4)$ & $376(28,6)$ & \\
\hline Mais de 55 anos & $1002(64,8)$ & $545(35,2)$ & \\
\hline
\end{tabular}

Tabela 5 - Estatísticas e testes $t$, relação entre a questão nº 16 e as afirmações $n^{\circ} 5,6$ e 7

\begin{tabular}{|c|c|c|c|}
\hline & \multicolumn{2}{|c|}{$\begin{array}{l}16 \text { - As terapêuticas não convencionais } \\
\text { deveriam ser incluídas no Serviço } \\
\text { Nacional de Saúde? }\end{array}$} & \multirow[b]{2}{*}{$p$} \\
\hline & $\begin{array}{c}\text { Não } \\
\text { M (DP) } \\
n=3138\end{array}$ & $\begin{array}{c}\operatorname{Sim} \\
\mathbf{M}(\mathrm{DP}) \\
\mathrm{n}=1196\end{array}$ & \\
\hline $\begin{array}{l}5 \text { - Os efeitos das terapêuticas não convencionais são essencialmente } \\
\text { resultado do efeito placebo. }\end{array}$ & $3,81(0,93)$ & $2,43(0,90)$ & $<0,001$ \\
\hline $\begin{array}{l}6 \text { - Os tratamentos não testados cientificamente de forma rigorosa } \\
\text { deverão ser desencorajados. }\end{array}$ & $4,27(0,83)$ & $3,28(1,07)$ & $<0,001$ \\
\hline $\begin{array}{l}7 \text { - As terapêuticas não convencionais incluem ideias e métodos que } \\
\text { poderiam ser utilizados de forma benéfica pela medicina convencional. }\end{array}$ & $2,95(1,04)$ & $4,07(0,67)$ & $<0,001$ \\
\hline
\end{tabular}

M: média; DP: desvio padrão

\section{DISCUSSÃO}

Tanto quanto sabemos, este é o primeiro estudo realizado em Portugal sobre as atitudes, conhecimentos e perspetivas dos médicos portugueses, de várias especialidades, em relação às TNC. Em 2010, Miranda ${ }^{25}$ abordou este tema estudando particularmente as perspetivas dos médicos portugueses de Medicina Geral e Familiar, pelo que não foi possível avaliar as convicções da classe médica no seu todo. Este trabalho representa um dos maiores estudos já realizados com a comunidade médica em Portugal, tendo o questionário sido respondido de forma completa por 4334 médicos, o que julgamos ser revelador do interesse dos médicos portugueses por este tema e da pertinência do debate do mesmo. Convém, no entanto, ter em conta que, apesar da sua dimensão, esta amostra corresponde a cerca de $8 \%$ do universo possível de respondentes e sendo 
Tabela 6 - Estatísticas e testes $t$, relação entre a questão n¹2 e as afirmações $n^{\circ} 1$ e 6

\section{2 - A competência em acupunctura médica da Ordem}

dos Médicos deveria no seu entender:

Permanecer disponível

Ser extinta

$M(D P)$

M (DP)

$(n=2973)$

$(n=1361)$

1 - A saúde física e mental é mantida por uma energia ou força vital.

3,10

3,75
1,12

1,03
2,00

4,54
1,07

0,69
$<0,001$

$<0,001$

6 - Os tratamentos não testados cientifice
rigorosa deverão ser desencorajados.

M: média; DP. desvio padrão

o questionário de resposta voluntária, as inferências para a população basearam-se numa amostra auto-selecionada e de conveniência. Além do mais, embora o questionário tenha sido construído com uma ferramenta informática que impedia a repetição do questionário no mesmo dispositivo, não foi possível evitar que uma mesma pessoa respondesse mais do que uma vez ao questionário em dispositivos eletrónicos diferentes. Deste modo, os resultados devem ser interpretados com os cuidados inerentes a estas limitações metodológicas.

Em relação ao conhecimento dos médicos portugueses acerca das TNC, demonstrou-se que a acupuntura é a terapêutica relativamente à qual existe perceção de maior grau de conhecimento por parte dos inquiridos, sendo a única com uma perceção de conhecimento médio superior ao ponto intermédio da escala medida. De um ponto vista geral, os médicos referem um grau de conhecimento baixo relativamente às terapêuticas não convencionais, sendo este facto corroborado pelas investigações anteriormente realizadas tanto em Portugal como internacionalmente, o que realça a necessidade de um maior investimento na formação médica no que diz respeito a esta temática. ${ }^{21,25}$

Os fatores que mais influenciam as convicções dos médicos relativamente às terapêuticas não convencionais são os "testemunhos de doentes", seguido de "opiniões de colegas profissionais de saúde", "revisões sistemáticas/metanálises". A importância dos relatos dos pacientes nas convicções dos médicos já tinha sido identificada por Miranda, o que permite destacar a importância da relação médico doente. ${ }^{25}$

Parte significativa dos médicos portugueses não se sentem confortáveis para conversar, argumentar e esclarecer os seus doentes a respeito das TNC. Patel et al,,$^{18}$ num estudo realizado no Ohio, já tinham identificado a carência de conhecimentos dos médicos relativamente a estas terapêuticas, o que evidencia mais uma vez a necessidade de aumentar os conhecimentos sobre a eficácia e a segurança das TNC de forma que os médicos se sintam mais preparados para aconselhar e orientar os seus doentes relativamente a estas terapêuticas. Para além disso, corroborando essa necessidade, identificou-se que a maioria dos médicos portugueses consideram que os currículos de formação médica deveriam incluir mais conteúdos sobre TNC.

Relativamente às atitudes face à referenciação para as TNC, estimou-se que $29,3 \%$ dos médicos portugueses já referenciaram um doente para um profissional de TNC. Em contraste, num estudo num centro médico norte americano apurou-se que $68 \%$ dos médicos inquiridos já tinham referenciado pacientes para este tipo de terapêuticas. ${ }^{17} \mathrm{~A}$ diferença observada relativamente a este ponto deve ser analisada tendo em consideração a forma como a estrutura de saúde norte americana está organizada, sendo que algumas destas terapêuticas estão enquadradas nos mesmos centros médicos da medicina convencional, o que poderá influenciar favoravelmente a percentagem de referenciação. No entanto, são necessários mais estudos de forma a entender de que forma se processa a referenciação e qual o impacto que isso tem nos diferentes países.

No respeitante às perspetivas dos médicos portugueses em relação às TNC, estimou-se que $68,6 \%$ dos médicos considera que a competência em acupuntura médica da Ordem dos Médicos deve permanecer disponível. Relativamente à inclusão das terapêuticas não convencionais no Serviço Nacional de Saúde, $72,4 \%$ dos médicos portugueses consideram que estas não devem fazer parte do SNS. A análise destes dados permite constatar que, maioritariamente, os médicos não são a favor do exercício integrado destas terapêuticas juntamente com a medicina convencional no seio do SNS como é sugerido na Lei de Bases da Saúde de 4 de setembro de 2019. ${ }^{29}$

Verificou-se a existência de associação entre o sexo e a idade dos médicos portugueses e as suas atitudes, conhecimentos e perspetivas relativamente às TNC. Wahner-Roedler et al/7 já tinham identificado que as mulheres referenciam mais os seus doentes para profissionais destas terapêuticas. A atualização dos currículos de formação médica ao longo das décadas poderá explicar a relevância da idade nas convicções dos médicos portugueses relativamente às TNC, no entanto são necessários mais estudos para perceber e clarificar o efeito destas variáveis.

\section{CONCLUSÃO}

Este estudo indica que os médicos portugueses sentem que os seus conhecimentos sobre terapêuticas não convencionais são diminutos. Parte significativa deles não se sente preparada para aconselhar apropriadamente os seus doentes neste âmbito. No entanto, a presença considerável de terapêuticas não convencionais na sociedade portuguesa, juntamente com o facto de não serem inócuas, torna impreterível que os médicos, como agentes fundamentais na comunicação com os seus doentes, tenham conhecimentos adequados sobre as TNC de forma a os poderem 
aconselhar e orientar. Este estudo realça, nesse sentido, a necessidade de incluir nos currículos de formação médica mais conteúdos sobre a eficácia e segurança das TNC. Do mesmo modo, poderá ser pertinente reforçar o ensino pré-graduado do método científico e dos princípios da medicina baseada na evidência, instrumentos essenciais para a melhor compreensão e avaliação da utilidade não só das TNC como dos diferentes métodos terapêuticos habituais.

Trabalhos futuros neste âmbito poderão debruçar-se de forma particular sobre as diferentes TNC presentemente regulamentadas, não só do ponto de vista dos profissionais, mas também dos seus utilizadores. A compreensão destes fenómenos será importante para uma melhor transmissão de informação à população por parte dos médicos envolvidos no seu cuidado.

\section{CONTRIBUTO DOS AUTORES}

CN, ABS, APM: Todos os autores contribuiram de igual forma para o desenho do estudo, recolha, armazenamento e análise de dados, elaboração do questionário, revisão e discussão dos resultados.

\section{REFERÊNCIAS}

1. National Center for Complementary and Integrative Health. Complementary, alternative, or integrative health: what's in a name? 2018 [consultado 2019 set 24]. Disponível em: https://nccih.nih.gov/ health/integrative-health.

2. Portugal. Decreto-Lei $n^{\circ} 45 / 2003$. Diário da República, I Série-A, nº 193 (2003/08/22). p.5391-2.

3. Portugal. Lei $n^{\circ}$ 71/2013. Diário da República, I Série, $n^{\circ} 168$ )2013/09/02).

4. Raposo VL. Complementary and alternative medicine, medical liability and the proper standard of care. Complement Ther Clin Pract. 2019;35:183-8.

5. Clarke TC, Black LI, Stussman BJ, Barnes PM, Nahin RL. Trends in the use of complementary health approaches among adults: United States, 2002-2012. Natl Health Stat Report. 2015;79:1-16.

6. Black LI, Clarke TC, Barnes PM, Stussman BJ, Nahin RL. Use of complementary health approaches among children aged 4-17 years in the United States: National Health Interview Survey, 2007-2012. Natl Health Stat Report. 2015;78:1-19.

7. Nahin RL, Barnes PM, Stussman BJ. Expenditures on complementary health approaches: United States, 2012. Natl Health Stat Report. 2016;95:1-12.

8. Nissen N, Schunder-Tatzber S, Weidenhammer W, Johannessen H. What attitudes and needs do citizens in Europe have in relation to complementary and alternative medicine? Complement Med Res. 2012;19:9-17.

9. Barros PP, Machado SR, Simões J de A. Portugal. Health system review. Health Syst Transit. 2011;13:1-156.

10. Mathie RT, Frye J, Fisher P. Homeopathic Oscillococcinum ${ }^{\circledR}$ for preventing and treating influenza and influenza-like illness. Cochrane Database Syst Rev. 2015;1:CD001957.

11. Smith C, Armour M, Lee M, Wang LQ, Hay P. Acupuncture for depression. Cochrane Database Syst Rev. 2018;3:CD004046.

12. Liu Y, Liu JP, Xia Y. Chinese herbal medicines for treating osteoporosis. Cochrane Database Syst Rev. 2014;3:CD005467.

13. Posadzki P, Ernst E. Spinal manipulation: an update of a systematic review of systematic reviews. N Z Med J. 2011;124:55-71.

14. Derry CJ, Derry S, McQuay HJ, Moore RA. Systematic review of systematic reviews of acupuncture published 1996-2005. Clin Med. 2006;6:381-6.

15. Sia $\mathrm{CH}$, Leow AS, Leong BS. Traumatic pneumothorax secondary to acupuncture needling. Cureus. 2018;10:1-6.

16. Ernst E. Fatalities after CAM: an overview. Br J Gen Pract. 2011;61: 404-5.

\section{PROTEÇÃO DE PESSOAS E ANIMAIS}

Os autores declaram que os procedimentos seguidos estavam de acordo com os regulamentos estabelecidos pelos responsáveis da Comissão de Investigação Clínica e Ética e de acordo com a Declaração de Helsínquia da Associação Médica Mundial actualizada em 2013.

\section{CONFIDENCIALIDADE DOS DADOS}

Os autores declaram ter seguido os protocolos do seu centro de trabalho acerca da publicação de dados.

\section{CONFLITOS DE INTERESSE}

Os autores declaram não ter qualquer conflito de interesse relativamente ao presente artigo.

\section{FONTES DE FINANCIAMENTO}

Os autores declaram que este estudo foi realizado sem recurso a subsídio ou bolsa.

17. Wahner-Roedler DL, Lee MC, Chon TY, Cha SS, Loehrer LL, Bauer BA Physicians' attitudes toward complementary and alternative medicine and their knowledge of specific therapies: 8-Year follow-up at an academic medical center. Complement Ther Clin Pract. 2014;20:54-60.

18. Patel SJ, Kemper KJ, Kitzmiller JP. Physician perspectives on education, training, and implementation of complementary and alternative medicine. Adv Med Educ Pract. 2017;8:499-503.

19. Olchowska-Kotala A, Barański J. Polish physicians' attitudes to complementary and alternative medicine. Complement Ther Med. 2016;27:51-7.

20. Maha N, Shaw A. Academic doctors' views of complementary and alternative medicine (CAM) and its role within the NHS: An exploratory qualitative study. BMC Complement Altern Med. 2007;7:1-11.

21. Bjerså K, Victorin ES, Olsén MF. Knowledge about complementary, alternative and integrative medicine (CAM) among registered health care providers in Swedish surgical care: a national survey among university hospitals. BMC Complement Altern Med. 2012;12:42.

22. Linde K, Alscher A, Friedrichs C, Wagenpfeil S, Karsch-Völk M, Schneider A. Belief in and use of complementary therapies among family physicians, internists and orthopaedists in Germany - crosssectional survey. Fam Pract. 2015;32:62-8

23. Brambila-Tapia A, Rios-Gonzalez B, Lopez-Barragan L, Saldaña-CruzA, Rodriguez-Vazquez K. Attitudes, knowledge, use, and recommendation of complementary and alternative medicine by health professionals in western Mexico. EXPLORE. 2016;12:180-7.

24. Wahner-Roedler DL, Vincent A, Elkin PL, Loehrer LL, Cha SS, Bauer BA Physicians' attitudes toward complementary and alternative medicine and their knowledge of specific therapies: a survey at an academic medical center. Evid Based Complement Alternat Med. 2006;3:495-501.

25. Miranda A. Terapêuticas não convencionais: perspectivas dos médicos de medicina geral e familiar. Covilhã: Faculdade de Ciências da Saúde, Universidade da Beira Interior; 2010.

26. Lie D, Boker J. Development and validation of the CAM Health Belief Questionnaire (CHBQ) and CAM use and attitudes amongst medical students. BMC Med Educ. 2004;4:1-9.

27. Sullivan GM, Artino Jr AR. Analyzing and interpreting data from likerttype scales. J Grad Med Educ. 2013;5:541-2.

28. Marôco J. Análise Estatística com o SPSS Statistics. $5^{a}$ ed. Coimbra: Edições Report Number; 2011

29. Portugal. Lei $n^{\circ}$ 95/2019. Diário da República, I Série, $n^{\circ} 169$ (2019/09/04). p.55-66. 